\title{
ABUNDANCES IN MAGELLANIC CLOUD PLANETARY NEBULAE
}

\author{
M.J. BARLOW \\ Department of Physics \& Astronomy \\ University College London \\ Gower St, London WC1E 6BT.
}

\begin{abstract}
The enhanced nitrogen abundances in planetary nebulae (PN) show the effects of CNcycle processing at the time of the first dredge-up, while their very enhanced carbon abundances show the effects of the third dredge-up. The efficiency of both the first and third dredge-ups increases with decreasing initial metallicity. ON-cycle depletion of oxygen via the second dredge-up does not appear to have been significant in LMC Type I planetary nebulae (those with N/O $>0.5$ ) - these nebulae have experienced the third dredge-up and envelope-burning, giving enhanced $\mathrm{C}+\mathrm{N}$ abundances. The LMC Type I PN show enhanced Ne/O ratios. Enrichment rates in nitrogen and carbon by PN are estimated for the ISM $\mathrm{s}$ of the Magellanic Clouds.
\end{abstract}

\section{Introduction}

In this article, I will concentrate on the work carried out in the three years since the review of Magellanic Cloud planetary nebula research given in 1987 at the Mexico City Symposium on PN (Barlow 1989). Earlier reviews of work on Magellanic Cloud PN were given by Peimbert (1984) and Jacoby (1983).

The analysis of PN spectra provides one with a means of determining accurate elemental abundances for material that was once part of the envelopes of stars that recently reached (and left) the tip of the asymptotic giant branch (AGB: see the review by Iben \& Renzini 1983). As such, the abundances derived for PN are more appropriate to use when estimating elemental enrichment rates for the interstellar medium (ISM), since abundances derived for individual AGB star envelopes represent snapshots during their evolution up the AGB.

The emission line analysis of photoionized nebulae is a fully developed and well understood branch of astrophysics, so that nebular abundances can be determined to quite a high degree of accuracy, given good quality spectra. After correction of the relative line intensities for interstellar reddening, through comparison of observed to theoretical recombination line intensity ratios, line flux ratios from certain species (such as [O III] and [O II]) are used to estimate nebular electron temperatures and densities. In conjunction with the appropriate line fluxes relative to hydrogen recombination lines, the electron temperatures and densities are then employed to determine ionic abundances relative to ionized hydrogen, where the observed emission lines are due to either collisional excitation, recombination or dielectronic recombination.

Spectrophotometry in the optical region can be used to derive the relative abundances of $\mathrm{He}, \mathrm{N}, \mathrm{O}, \mathrm{Ne}, \mathrm{S}$ and Ar: the abundances derived for the observed ionic stages often need to be multiplied by ionization correction factors (icf s) in order to allow for unseen ionic 
stages. Spectrophotometry in the ultraviolet is particularly important for the derivation of carbon abundances, as there are strong C II], C III] and C IV lines there. In addition, further ionic stages of $\mathrm{N}, \mathrm{O}$ and $\mathrm{Ne}$ can be observed in the UV, lessening the need to use icf s. However, for the same nebulae, comparisons between nitrogen abundances based on (a) large icf $\mathrm{s}$ applied to $\mathrm{N}^{+}$abundances from optical lines and (b) total abundances based on $\mathrm{N}^{2+}, \mathrm{N}^{3+}$ and $\mathrm{N}^{4+}$ lines observed in the ultraviolet, have shown good agreement (e.g. Barker 1984).

During their evolution before becoming planetary nebulae, stars may experience a number of 'dredge-up' episodes which can significantly alter the relative abundances in their outer envelopes. It is worth recalling these episodes (a full description is given by Iben \& Renzini 1983 and Renzini \& Voli 1981):

First dredge-up: this is experienced by all low and intermediate mass stars (i.e. those with masses less than about 8-9 $\mathrm{M}_{\odot}$ ) at the start of the first ascent of the red giant branch. It enriches the stellar envelope with the products of the $\mathrm{CN}$ cycle, i.e. helium and nitrogen, and can result in a significant decrease in the surface carbon abundance, depending on the initial metallicity (Becker \& Iben 1980).

Second dredge-up: this is experienced only by intermediate mass stars $\left(M>2.5 M_{\odot}\right)$ at the start of the ascent of the AGB and brings to the surface the products of the CNO cycle, i.e. helium and nitrogen. Carbon and oxygen are depleted in the dredged-up material, although the resulting surface abundance of oxygen is not predicted to be depleted significantly (by $30 \%$ at most, Becker \& Iben 1980).

Neither the first nor second dredge-ups brings about a change in the total $\mathrm{C}+\mathrm{N}+\mathrm{O}$ abundance.

Third dredge-up: this results from thermal pulses in the shell helium-burning zone during the ascent of the AGB by low and intermediate mass stars. It brings helium and carbon to the surface, plus s-process elements. Given a sufficient number of thermal pulses during its ascent of the AGB, the star may eventually be turned into a carbon star $(C / O>1)$. However, for stars sufficiently massive that the base of their hydrogen zone is hot enough for the CN cycle to operate, the transition to a carbon star may never occur, due to the conversion of the dredged-up carbon to nitrogen (this process has been dubbed 'envelopeburning'). Like the carbon, this nitrogen would then be a primary nucleosynthesis product - the total $\mathrm{C}+\mathrm{N}+\mathrm{O}$ abundance is increased as a result of the third dredge-up.

Finally, for the purposes of this article, a Type I PN will be defined as any planetary nebula which has $\mathrm{N} / \mathrm{O}>0.5$ by number (this is related to the definition of Galactic Type Is by Peimbert \& Torres-Peimbert 1983). Galactic Type I PN are usually bipolar in morphology and are believed to have originated from progenitor stars more massive than those of the majority of PN. For example, the bipolar Type I PN NGC 2818 is a member of the open cluster of the same name, with a main sequence turn-off mass of $>2.5 \mathrm{M}_{\odot}$ (Dufour 1984a).

In Section 2, the abundance patterns in non-Type I PN are discussed, while Type I PN are dealt with in Section 3. In Section 4, some estimates are made for the enrichment in nitrogen and carbon of the interstellar media of the Magellanic Clouds by planetary nebulae.

\section{Abundance Surveys}

During the past three years the results of a number of abundance surveys of Magellanic 
Cloud PN have been published. The optical survey of Monk et al. (1988) presented results for 71 nebulae, while the optical survey of Boroson \& Liebert (1989; analysed by Henry et al. 1989) presented data for the 29 bona fide $P N$ in the deep sample of Jacoby (1980). Aller et al. (1987) analysed both IUE and optical spectrophotometric data for 12 Magellanic Cloud PN, while Walton et al. (1990) have presented the results obtained from an analysis of $I U E$ and optical spectra for 38 nebulae.

\subsection{THE O-NE RELATION}

Henry et al. (1989) noted that a plot of neon versus oxygen abundances for their 29 Magellanic Cloud PN, together with 75 Galactic PN, produced a striking correlation. Henry (1989) enlarged the total sample to $171 \mathrm{PN}$ in the Galaxy, Magellanic Clouds and M 31 by re-analysing previously published data in a homogeneous fashion. The strong correlation was confirmed, a mean $\mathrm{Ne} / \mathrm{O}$ ratio of $0.17 \pm 0.06$ by number being derived for the $\mathrm{PN}$. The data for Magellanic Cloud PN were crucial for establishing this result. The PN Ne/O relation was found to be very similar to that found for $\mathrm{H}$ II regions in the same galaxies.

Henry noted that both oxygen and neon are predicted to be synthesised significantly only by massive stars $\left(\sim 20 \mathrm{M}_{\odot}\right)$ and that since the $\mathrm{Ne} / \mathrm{O}$ ratio predicted by the models varied as a function of initial stellar mass, the observed $\mathrm{Ne} / \mathrm{O}$ ratio could in principle yield constraints on the IMF for massive stars. It was noted, however, that uncertainties in the models, e.g. semi-convection, convective overshooting, and the ${ }^{12} \mathrm{C}(\alpha, \gamma){ }^{16} \mathrm{O}$ reaction rate, currently prevented the use of the $\mathrm{Ne} / \mathrm{O}$ ratio as an IMF diagnostic.

In Section 3, we will return to the question of $\mathrm{Ne} / \mathrm{O}$ ratios, in the context of Type I PN.

\subsection{NITROGEN ABUNDANCES}

Monk et al. (1988) showed that the nitrogen abundances in non-Type I PN were consistent with the conversion of initial carbon to nitrogen by the $\mathrm{CN}$ cycle, operating in the progenitor stars at the time of the first dredge-up. Becker \& Iben (1980) had predicted that, following the first dredge-up, about one third of the initial surface carbon should be converted to nitrogen, for the case of low mass stars of solar composition, while for low-mass stars of Population II composition, nearly $100 \%$ of the initial surface carbon should be converted to nitrogen. The results of Monk et al. were in good agreement with these predictions, their derived carbon conversion rates being $20 \%, 70 \%$ and $100 \%$ for the Galaxy, LMC and SMC, respectively.

The higher S/N data of Walton et al. (1990) have allowed the first dredge-up carbon conversion fractions to be evaluated more accurately. Table 1 presents $\mathrm{C}, \mathrm{N}, \mathrm{O}$ and $\mathrm{Ne}$ abundances for H II regions and planetary nebulae in the SMC, LMC and Galaxy, along with the solar abundances for these elements given by Grevesse \& Anders (1989). It is assumed that the initial abundances in the PN progenitor stars were the same as those observed in the $\mathrm{H}$ II regions of the same galaxy. The fourth column of Table 1 lists (in bold-face) the total $\mathrm{C}+\mathrm{N}$ abundance in the $\mathrm{H}$ II regions of each galaxy, which can be compared with the $\mathrm{N}$ abundances of the non-Type I PN, also given in bold-face. The comparison implies carbon-to-nitrogen conversion rates by the first dredge-up of $20 \%, 50 \%$ and $100 \%$ for the Galaxy, LMC and SMC, respectively. It is interesting that these differing conversion rates have led to almost the same final $\mathrm{N} / \mathrm{O}$ ratio for the non-Type I PN in each galaxy, $\log \mathrm{N} / \mathrm{O} \sim-0.74$ (Table 2). 


\section{Table 1: $\mathrm{C}, \mathrm{N}, \mathrm{O}$, and Ne abundances}

MC PN: Walton et al. (1990). H II: Pagel et al. (1978), Dufour (1984b) Galactic PN: Torres-Peimbert \& Peimbert (1977), Aller \& Czyzak (1983)

Mean logarithmic abundances, $\mathrm{H}=12.0$

$$
\begin{array}{lllll}
\mathrm{C} & \mathrm{N} & \mathrm{C}+\mathrm{N} & \mathrm{O} & \mathrm{Ne}
\end{array}
$$

$\begin{array}{lccccc}\text { SMC H II } & 7.16 \pm 0.04 & 6.46 \pm 0.12 & \mathbf{7 . 2 4} \pm \mathbf{0 . 0 5} & 8.02 \pm 0.08 & 7.22 \pm 0.12 \\ \text { 14 Non Type I } & 8.87 \pm 0.27 & \mathbf{7 . 4 4} \pm \mathbf{0 . 1 7} & & 8.20 \pm 0.15 & 7.42 \pm 0.24 \\ \text { 3 Type I } & 6.94 \pm 0.29 & 7.77 \pm 0.16 & & 7.61 \pm 0.13 & 7.12 \pm 0.08 \\ \text { All SMC PN } & 8.79 \pm 0.30 & 7.51 \pm 0.16 & & 8.15 \pm 0.16 & 7.38 \pm 0.24 \\ & & & & & \\ \text { LMC H II } & 7.90 \pm 0.15 & 6.97 \pm 0.10 & \mathbf{7 . 9 5} \pm \mathbf{0 . 1 5} & 8.43 \pm 0.08 & 7.64 \pm 0.10 \\ \text { 12 Non Type I } & 8.78 \pm 0.34 & \mathbf{7 . 6 8} \pm \mathbf{0 . 2 6} & & 8.41 \pm 0.18 & 7.61 \pm 0.25 \\ \text { 5 Type I } & 8.45 \pm 0.50 & 8.36 \pm 0.12 & & 8.41 \pm 0.20 & 7.80 \pm 0.19 \\ \text { All LMC PN } & 8.70 \pm 0.35 & 7.98 \pm 0.24 & & 8.41 \pm 0.17 & 7.68 \pm 0.19 \\ & & & & & \\ \text { Gal H II } & 8.46 \pm 0.2 & 7.57 \pm 0.33 & \mathbf{8 . 5 1} \pm \mathbf{0 . 3 0} & 8.70 \pm 0.25 & 7.90 \pm 0.17 \\ \text { Gal PN } & 8.76 \pm 0.3 & \mathbf{7 . 9 6} \pm \mathbf{0 . 3 2} & & 8.68 \pm 0.14 & 7.98 \pm 0.20 \\ \text { Solar } & 8.56 \pm 0.04 & 8.05 \pm 0.04 & & 8.93 \pm 0.03 & 8.09 \pm 0.10\end{array}$

Table 2: Abundance ratios

$$
\log \mathrm{C} / \mathrm{O} \quad \log \mathrm{N} / \mathrm{O} \quad \log \mathrm{Ne} / \mathrm{O}
$$

$\begin{array}{lccc}\text { SMC H II } & -0.86 \pm 0.08 & -1.57 \pm 0.10 & -0.80 \pm 0.10 \\ \text { 14 Non-Type I } & +0.67 \pm 0.20 & -0.76 \pm 0.02 & -0.79 \pm 0.02 \\ \text { 3 Type I } & -0.67 \pm 0.20 & +0.17 \pm 0.02 & -0.48 \pm 0.01 \\ \text { All SMC PN } & +0.64 \pm 0.20 & -0.64 \pm 0.02 & -0.77 \pm 0.02 \\ & & & \\ \text { LMC H II } & -0.53 \pm 0.12 & -1.51 \pm 0.10 & -0.79 \pm 0.12 \\ \text { 12 Non-Type I } & +0.36 \pm 0.07 & -0.73 \pm 0.19 & -0.80 \pm 0.03 \\ \text { 5 Type I } & +0.04 \pm 0.70 & -0.07 \pm 0.06 & -0.61 \pm 0.01 \\ \text { All LMC PN } & +0.29 \pm 0.22 & -0.44 \pm 0.28 & -0.74 \pm 0.02 \\ & & & \\ \text { Gal H II } & -0.24 \pm 0.2 & -1.13 \pm 0.29 & -0.80 \pm 0.21 \\ \text { Gal PN } & +0.08 \pm 0.2 & -0.72 \pm 0.23 & -0.70 \pm 0.17 \\ \text { Solar } & -0.37 \pm 0.04 & -0.88 \pm 0.04 & -0.84 \pm 0.07\end{array}$




\subsection{CARBON ABUNDANCES}

The ultraviolet and optical study of Aller et al. (1987) showed that among nine non-Type I PN in their sample, all but LMC N201 ( $\equiv$ P25; the most luminous PN in the Magellanic Clouds) had nebular $\mathrm{C} / \mathrm{O}$ ratios significantly larger than unity, so that their immediate progenitors must have been carbon stars; the high carbon abundances were ascribed to the third dredge-up.

The study of Walton et al. (1990) has increased by a factor of three the number of Magellanic Cloud PN for which both UV and optical data have been analysed, allowing some further inferences to be drawn. Their results (Tables 1 and 2) give mean $\mathrm{C} / \mathrm{O}$ number ratios of 4.7 and 2.3 for non-Type I nebulae in the SMC and LMC, respectively, significantly larger than the $\mathrm{C} / \mathrm{O}$ ratios found for most PN (and carbon stars) in our own Galaxy.

Walton et al. have estimated the surface carbon abundance enhancements due to the third dredge-up, by subtracting from the observed nebular carbon abundances the residual initial carbon abundances implied by the first dredge-up $\mathrm{C} \rightarrow \mathrm{N}$ conversion factors for $\mathrm{PN}$ in each galaxy (Section 2.2). It is found that $\Delta \mathrm{C} / \mathrm{H}=7.4 \times 10^{-4}, 5.6 \times 10^{-4}$ and $3.4 \times 10^{-4}$, by number, for the SMC, LMC and Galaxy, respectively. Since the mean nebular hydrogen masses and mean central star masses are the same for the SMC and LMC (Walton et al. 1990), one can conclude that the absolute amount of carbon that is dredged up per star increases from the Galaxy to the LMC to the SMC. This is in agreement with the theoretical results of Wood (1981), who found that the efficiency of the third dredge-up increases with decreasing metallicity.

The mean central star mass derived by Walton et al. (1990) for 14 nebulae having C/O $>1$ was $0.585 \pm 0.016 \mathrm{M}_{\odot}$. While evolutionary modelling of AGB stars has been successful in recent years in lowering the minimum core mass required for carbon star formation (see the review by Lattanzio, 1989), only a model by Boothroyd \& Sackmann (1988), which invoked a high mixing-length parameter of 3 , has been successful in producing a carbon star with this low a core mass.

\subsection{HELIUM ABUNDANCES}

From a recombination line analysis, Monk, Barlow \& Clegg (1988) derived $\mathrm{He} / \mathrm{H}$ ratios for 13 SMC PN and $32 \mathrm{LMC} \mathrm{PN}$, taking into account the contribution by collisional excitation to the observed He I line strengths, using the correction formulae of Clegg (1987). They found that without any correction for $\mathrm{He} \mathrm{I}$ collisional excitation, the $\mathrm{He} / \mathrm{H}$ number ratios derived for $\mathrm{PN}$ were significantly higher than those for $\mathrm{H}$ II regions in the same galaxy (e.g. 0.105 vs. 0.083 in the LMC), whereas after correction for collisional excitation of He I, the derived ratios for PN were only slightly higher than those for H II regions (e.g. $0.087 \pm 0.008 v s$. $0.082 \pm 0.004$ in the LMC). This is certainly consistent with predictions for the first dredge-up, which should give a surface helium enhancement $\Delta \mathrm{He} / \mathrm{H} \sim 0.005$, by number, for an initial mass of $1.5 \mathrm{M}_{\odot}$ (Becker \& Iben 1980). However, the increase in surface $\mathrm{He} / \mathrm{H}$ as a result of the third dredge-up should be comparable to or larger than that produced by the first dredge-up. The results of Boothroyd \& Sackmann (1988) for the third dredge-up predict $\mathrm{He} / \mathrm{C}=10$, by number, in the dredged-up material. From the values of surface $\Delta \mathrm{C} / \mathrm{H}$ derived in Section 2.3, one therefore predicts helium enhancements due to the third dredge-up equivalent to $\Delta \mathrm{He} / \mathrm{H}=0.0074,0.0056$ and 0.0034 for the SMC, LMC and Galaxy, respectively. It is therefore not clear whether the helium enhancements 
derived by Monk et al. (1988) are consistent with the predictions of these models.

A further complication in the interpretation of derived helium abundances is that Peimbert \& Torres-Peimbert (1987) have suggested that the contribution of collisional excitation to the He I lines is only 50-60\% of that given by the formulae of Clegg (1987), which would then imply slightly higher $\mathrm{He} / \mathrm{H}$ ratios than those derived by Monk et al., who used the full corrections. It would appear that a proper comparison of the observed $\mathrm{He} / \mathrm{H}$ ratios in PN with theoretical predictions will not be possible until the exact collisional corrections appropriate for the He I lines have been determined.

\section{Type I Planetary Nebulae}

Aller et al. (1987) found that the one SMC and the two LMC. Type I PN in their sample exhibited low $\mathrm{C} / \mathrm{O}$ number ratios. They also derived low oxygen abundances for these objects and attributed the low carbon and oxygen abundances to the effects of the second dredge-up. Henry (1990) also invoked oxygen depletion by the ON cycle, presumably during the second dredge-up, to explain Type I abundance patterns.

The results of Walton et al. (1990) for three SMC Type I planetary nebulae (Table 1) indicate that the abundances of all heavy elements in them are significantly lower than in other PN or H II regions in the SMC, making it less easy to interpret their abundance patterns. However, Walton et al.'s results for five Type I nebulae in the LMC are straightforward to interpret. The mean oxygen abundance was found to be the same as for non-Type I $\mathrm{PN}$ and $\mathrm{H}$ II regions in the LMC (Table 2), indicating that $\mathrm{ON}$-cycle depletion and the second dredge-up did not significantly affect the surface oxygen abundances in the progenitors of the Type I nebulae. The main reason for the difference between the conclusions of Walton et al. and Aller et al. (1987) is that larger abundances are estimated for high ionization stages by Walton et al. than by Aller et al..

Did the third dredge-up operate in the Type I nebula progenitor stars? Walton et al. found that the nitrogen abundance alone in the LMC Type Is exceeded the $\mathrm{H}$ II region $\mathrm{C}+\mathrm{N}$ abundance by $0.2-0.5$ dex, while the Type I $\mathrm{C}+\mathrm{N}$ abundances were $0.35-1.05$ dex larger than those of $\mathrm{H}$ II regions, implying the exposure of primary nucleosynthesis products via the third dredge-up and envelope-burning. Figure 1 of Walton et al. (1990) shows that two of the LMC Type I nebulae (N28 and N122) have C/O $>1$, while the other three (N66, N97 and $\mathrm{N} 102$ ) have $\mathrm{C} / \mathrm{O}<1$. The sequence of decreasing $\mathrm{C} / \mathrm{O}$ ratio among Type I nebulae may represent a sequence of increasing core mass and and increasing efficiency for the conversion of dredged-up carbon to nitrogen by envelope-burning.

It has been noted (Clegg 1989; Henry 1989) that Type I planetary nebulae may have larger Ne/O ratios than other PN. This is confirmed by the results of Walton et al. (1990). Their Ne/O abundance ratios (Table 2) have a much smaller scatter than do the $\mathrm{O} / \mathrm{H}$ or $\mathrm{Ne} / \mathrm{H}$ ratios (Table 1 ). The LMC Type I PN have Ne/O ratios which are $0.19 \pm 0.03$ dex larger than those of non-Type I nebulae (the enhancement is even larger in the SMC Type I s). Since the mean $\mathrm{O} / \mathrm{H}$ abundance found for the LMC Type I nebulae is the same as that found for the non-Type I s, an enhancement of neon seems to be implied. A mechanism for this, in the helium-burning zone, is successive $\alpha$-particle captures onto ${ }^{14} \mathrm{~N}$ to give ${ }^{22} \mathrm{Ne}$ (Clegg 1989). However, if this mechanism operated solely on the nitrogen produced by CNO re-cycling of the initial stellar $\mathrm{C}, \mathrm{N}$ and $\mathrm{O}$, then the maximum enhancement of neon that could result from the third dredge-up would be only $0.07-0.08 \mathrm{dex}$ in the LMC and SMC (obtained by combining the surface carbon enhancements due to the 3rd dredge-up, 
given in Section 2.2, with the predictions of Boothroyd \& Sackmann (1988) for the carbon fraction in the dredged-up material). This seems insufficient to account for the observed neon enhancements in the Type I nebulae. The most likely possibility seems to be that some of the primary nitrogen produced by $\mathrm{CN}$-cycle envelope-burning of dredged-up carbon is mixed back down to the helium envelope, where it is converted to ${ }^{22} \mathrm{Ne}$. Since the neon and oxygen abundances derived for Type I PN can be very dependent on the icf s adopted for the highest stages of ionization, it is clear that further work on this topic is desirable.

\section{Enrichment of the ISM by Planetary Nebulae}

We have seen how nitrogen and carbon are significantly enriched in the envelopes of planetary nebulae, so it is of interest to estimate the contribution by $\mathrm{PN}$ to the enrichment of the interstellar media of the Magellanic Clouds in these elements. To do this, one can combine the nitrogen and carbon surface abundance enhancements for PN (Sections 2.2, $2.3 \& 3$ ) with the following parameters: (a) a mean nebular hydrogen mass of $0.217 \mathrm{M}_{\odot}$ for non-Type I PN in both the SMC and LMC, $0.28 \mathrm{M}_{\odot}$ for SMC Type I PN, and $0.36 \mathrm{M}_{\odot}$ for LMC Type I PN (Walton et al. 1990), with Type I nebulae representing $18 \%$ of all PN in the Magellanic Clouds (Monk et al. 1988); (b) an observable lifetime of 20,000 years for a planetary nebula; and (c) a total of $120 \mathrm{PN}$ in the SMC and $660 \mathrm{PN}$ in the LMC, based on the analysis by Boroson \& Liebert (1989) of Jacoby's (1980) deep sample.

One then finds ISM nitrogen enrichment rates due to PN of $4.5 \times 10^{-8}$ and $6.8 \times 10^{-7} \mathrm{M}_{\odot}$ $\mathrm{yr}^{-1}$ for the SMC and LMC, of which Type I PN contribute $38 \%$ and $67 \%$, respectively. Similarly, the PN carbon enrichment rates for the ISM s of the SMC and LMC are found to be $7.7 \times 10^{-7}$ and $3.7 \times 10^{-6} \mathrm{M}_{\odot} \mathrm{yr}^{-1}$. With $5 \times 10^{8} \mathrm{M}_{\odot}$ of $\mathrm{H} \mathrm{I}$ in each Cloud (Westerlund 1990), these enrichment rates imply characteristic times for doubling the ISM nitrogen abundances to their current values (Table 1) of $1.6 \times 10^{10}$ and $3.5 \times 10^{9}$ years, for the SMC and LMC. For carbon, these characteristic timescales become $4.6 \times 10^{9}$ and $5.5 \times 10^{9}$ years for the SMC and LMC.

These enrichment timescales, which are comparable to the mean ages estimated for the PN populations of the Magellanic Clouds (Meatheringham et al. 1988), indicate that the low and intermediate mass Magellanic Cloud stars that evolve into planetary nebulae are probably the dominant sources of enrichment of their ISM $s$ in carbon, and possibly in nitrogen as well. This is consistent with the observation that, relative to our own Galaxy, the H II regions of the Magellanic Clouds are much more deficient in carbon and nitrogen than in oxygen or neon (Table 1), since the latter elements are thought to be due to prompt enrichment by massive stars.

\section{References}

Aller, L. H. \& Czyzak, S. J., 1983. Astrophys. J. Suppl., 51, 211.

Aller, L. H., Keyes, C. D., Maran, S. P., Gull, T. R., Michalitsianos, A. G. \& Stecher, T. P., 1987. Astrophys. J., 320, 159.

Barker, T., 1984. Astrophys. J., 284, 589.

Barlow, M. J., 1989. IAU Symp. No. 131, p. 319, ed. Torres-Peimbert, S., Kluwer, Dordrecht, Holland.

Becker, S. A. \& Iben, I., Jr, 1980. Astrophys. J., 237, 111. 
Boothroyd, A. I. \& Sackmann, I.-J., 1988. Astrophys. J., 328, 653.

Boroson, T. A. \& Liebert, J., 1989. Astrophys. J., 339, 844.

Clegg, R. E. S., 1987. Mon. Not. R. astr. Soc., 229, 31P.

Clegg, R. E. S., 1989. IAU Symp. No. 131, p. 139, ed. Torres-Peimbert, S., Kluwer, Dordrecht, Holland.

Dufour, R. J., 1984a. Astrophys. J., 287, 341.

Dufour, R. J., 1984b. IAU Symp. No. 108, p. 353, eds. van den Bergh, S. \& de Boer, K. S., Reidel, Dordrecht, Holland.

Grevesse, N. \& Anders, E., 1989. Cosmic Abundances of Matter, AIP Conf. Proc. 183, p. 1, ed. Waddington, J., AIP, New York, USA.

Henry, R. B. C., 1989. Mon. Not. R. astr. Soc., $241,453$.

Henry, R. B. C., 1990. Astrophys. J., 356, 229.

Henry, R. B. C., Liebert, J. \& Boroson, T. A., 1989. Astrophys. J., 339, 872.

Iben, I., Jr \& Renzini, A., 1983. Ann. Rev. Astr. Astrophys., 21, 271.

Jacoby, G. H., 1980. Astrophys. J. Suppl., 42, 1.

Jacoby, G. H., 1983. IAU Symp. No. 103, p. 427, ed. Flower, D. R., Reidel, Dordrecht, Holland.

Lattanzio, J., 1989. Evolution of Peculiar Red Giant Stars, IAU Colloq. No. 106, p. 161, eds. Johnson, H. R. \& Zuckerman, B., Cambridge Univ. Press, Cambridge, UK.

Meatheringham, S. J., Dopita, M. A., Ford, H. C. \& Webster, B. L., 1988. Astrophys. J., 327, 651 .

Monk, D. J., Barlow, M. J. \& Clegg, R. E. S., 1988. Mon. not. R. astr. Soc., 234, 583.

Pagel, B. E. J., Edmunds, M. G., Fosbury, R. A. E. \& Webster, B. L., 1978. Mon. Not. R. astr. Soc., 184, 569.

Peimbert, M., 1984. IAU Symp. No. 108, p. 363, eds. van den Bergh, S. \& de Boer, K. S., Reidel, Dordrecht, Holland.

Peimbert, M. \& Torres-Peimbert, S., 1983. IAU Symp. No. 103, p. 233, ed. Flower, D. R., Reidel, Dordrecht, Holland.

Peimbert, M. \& Torres-Peimbert, S., 1987. Rev. Mex. Astr. Astrof., 15, 117.

Renzini, A. \& Voli, M., 1981. Astr. Astrophys., 94, 175.

Torres-Peimbert, S. \& Peimbert, M., 1977. Rev. Mex. Astr. Astrof., 2, 181.

Walton, N. A. , Barlow, M. J., Monk, D. J. \& Clegg, R. E. S., 1990. These Proceedings.

Westerlund, B. E., 1990. Astr. Astrophys. Rev., 2, 29.

Wood, P. R., 1981. Physical Processes in Red Giants, p. 205, eds. Iben, I. Jr \& Renzini, A., Reidel, Dordrecht, Holland. 